\title{
Evolutionary Approach to jointly resolve the Power and the Capacity Optimization problems in the multi-user OFDMA Systems
}

\author{
Ndiaye Abdourahmane, Ouya Samuel and Mendy Gervais \\ Ecole Supérieure Polytechnique \\ Université Cheikh Anta DIOP \\ Dakar, Sénégal \\ BP:5085, Dakar-Fann, Sénégal
}

\begin{abstract}
This paper deals with the problem of resources allocation in the downlink of the radio mobile systems. The allocation of resources is established in the context of multi-path effect and Doppler effect. These phenomena cause randomly variations of the channel and make difficult the allocation of the resources. Therefore, the problem of resources allocation is a high non linear optimization problem. Thus, we propose an implementation of genetic algorithms approach to increase the total throughput of users while minimizing the consumption of the total power of the base station. Like the evolutionary algorithms approach, this method is characterized by its robustness, permitting to efficiently solve a non linear optimization problem.
\end{abstract}

Keywords-Cellular Network; OFDMA; Resources Allocation; Rate Adaptive; Margin Adaptive; Genetic Algorithms

\section{INTRODUCTION}

Today, the requirements of the users's data rate are becoming more and more increasing; which led to the adoption of efficient resources allocation methods in order to improve the QoS in mobile radio communications. These resources management methods form the basis of the fourth generation of mobile networks systems.

The 4th generation of mobile systems such as LTEAdvanced, will offer high data rate services and large capacity of users. The major change of these systems is the access method OFDMA (Orthogonal Frequency Division Multiplexing Access) which significantly increases the capacity by the improvement of the signal-to-noise ratio obtained with an adaptive resources allocation [13].

The access method OFDMA is base on the transmission technique OFDM(Orthogonal Frequency Division Multiplexing) which consist of the frequency multiplexing of modulated orthogonal sub-carriers. The method OFDM is very suitable for the variations of the channel caused in particular by the multi-path phenomenon and the Doppler effect.

The principle of the modulation OFDM is to split the available bandwidth into many flat sub-channels which can be attributed independently to users [13]. In addition, the quantity of bits transmitted on each sub-channel can be adapted according to the channel gain level in order to obtain a considerable power gain with the required bit error rate [2].
The independence of the OFDM sub-carriers permits an efficient resources allocation. In the context of channel characterized frequency selectivity and time variation, the efficient allocation of resources consists in affecting the sub-carriers to users taking into account the specific channel gain for each users. In preference, the sub-carriers are allocated to users with highest channel gain while satisfying the required data rate for each user.

In this paper, we propose an implementation of genetic algorithms for solving the rate-adaptive and the margin-adaptive problems, subject to the available total power in the base station and the minimal required users's data rate. We consider the downlink of the OFDMA based-systems and we take into account the propagation path-loss so as to guarantee effective throughput to users in reception.

In Section 2, the related work about resources allocation is described. In Section 3, the modeling system and the formulation of the optimization problem are presented. In Section 4, the principle of genetic algorithms is given and in Section 5, the proposed algorithm is described. Finally, in Section 6 , we compare the results of the proposed algorithm to others algorithms.

\section{RELATED WORK}

The algorithmic approach for the resources allocation problem is introduced by Ibaraki et al. [7]. Many algorithms are proposed to solve the problem of resources allocation. There are the iterative methods and the heuristic methods. Among the iterative approaches, there are the water-filling algorithm which solves the problem of power allocation in single user case. The work proposed by Tu et al. [16] is based on the waterfilling approach. The resolution of the resources allocation problem in multi-users case is proposed in [3][5]. About the heuristic methods, Zhou et al. [17], Ahmadi et al. [1] and Reddy et al. [12] proposed the genetic algorithms approach for the resources allocation problem.

In several works cited above, some aspects are not taken into account for instance the path-loss which is a very determinant parameter in order to have the realist data rate for users at the reception. In addition, the model of channel gain chosen in this work, takes simultaneously into account the 
multi-path and the Doppler effects. Thus, in this paper we propose an implementation of genetic algorithms for solving the rate-adaptive problem, subject to the available total power in the base station. In this work, we consider the downlink of the OFDMA based-systems and we take into account the propagation path-loss so as to guarantee effective throughput to users in reception.

\section{System MOdELING AND Formulation OF THE PROBLEM}

\section{A. System modeling}

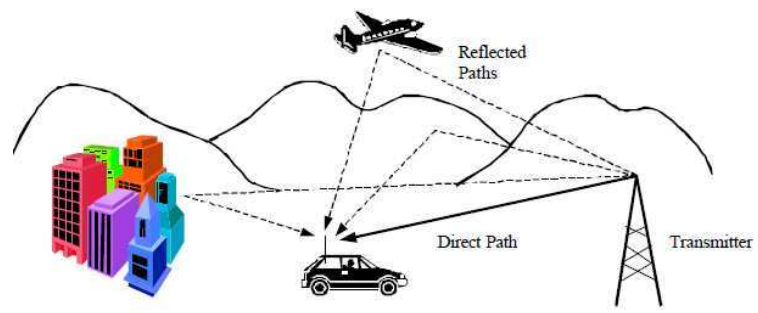

Fig. 1. Multi-path channel with Doppler effect

In this work, we study the allocation of resources in the downlink of mobile network based on the OFDMA access method (Orthogonal Frequency Division Multiplexing Access). We consider a mono-cellular system with multiple access and we suppose that the users are uniformly distributed in the cell. The channel of transmission is assumed to be characterized by the multi-path and the Doppler effects (see figure 1). These phenomena lead respectively to the frequency selectivity and the time-variant of the channel [14]. The time-variant of the radio channel propagation is caused by the movement of the transmitter and/or the receiver. Thus, the channel is nonstationary, and this gain varies in the frequency and in the time domain according to each user.

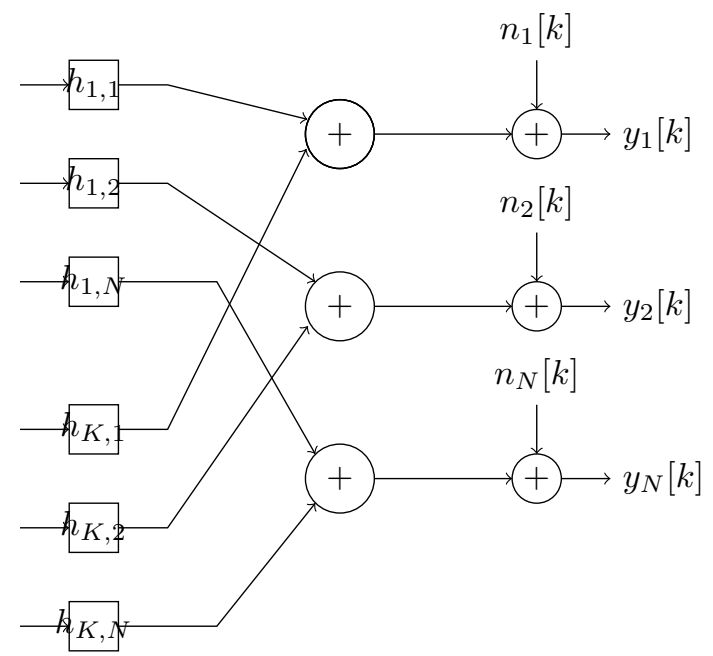

Figure 2: Multi-user OFDM systems

The base station (transmitter) is considered to be fixed and the users are assumed to be in mobility. Therefore, the Doppler effect depends on the speed of users and the frequency used in the transmission. Let's consider $v$ and $f_{0}$ respectively the speed of the users and the frequency of the transmitted signal. The maximum Doppler shift is given by:

$$
f d_{\max }=\frac{v}{c} f_{0}
$$

where $c$ is the celerity of the light.

For an angle $\alpha$ between the direction of transmission and the movement of user, the Doppler frequency shift is defined by:

$$
f d=f d_{\max } \cos (\alpha)
$$

In the case of Doppler effect, the received signal is characterized by spatial interference pattern which causes, when the user is moving in this pattern, many fluctuations of the amplitude of the signal [14]. The time-variant of the channel is characterized by the time of correlation $\tau_{c o r}=1 / f d_{\max }$. The frequency selectivity corresponds to a variation of channel gain depending on the transmission frequency; therefore for a certain frequency, the attenuation can be very important and entail the loss of the transmitted signal. The coherence bandwidth $B_{c o h}=1 / \mathrm{rms}$ is the characteristic parameter of the frequency selectivity of the channel, where rms is the maximum root mean square of the path delay.

\section{B. Formulation of the margin adaptive's problem}

The problem of margin adaptive deals with the minimization of the total power consumption in the downlink of the cellular system while the request of data rate for users is achieved. The channel transmission is assumed to be characterized by time variation and frequency selectivity. Therefore, the channel presents for users, an specified channel gain which depends on the position et the velocity of the users.

Let's consider $H_{k, n}$ the channel gain of $k t h$ user for $n t h$ sub-carrier. The required power is given by[11]:

$$
p_{k, n}=\frac{f\left(c_{k, n}\right)}{H_{k, n}^{2}}
$$

where

$$
f\left(c_{k, n}\right)=\frac{N_{0}}{3}\left(2^{c_{k, n}}-1\right)\left[Q^{-1}\left(\frac{B E R_{k}}{4}\right)\right]^{2}
$$

$c_{k, n}$ is the number of bits for kth user on the nth sub-carrier. $N_{0}$ is the power spectral density of noise.

$B E R_{k}$ is the bits error rate of $k t h$ user.

$Q(x)=\operatorname{erfc}(x)$ is the complementary error function.

We note that when the channel gain $H_{k, n}$ increases the power required decreases. Thus the $n t h$ sub-carrier will be allocated in preference to the kth user with least power until the required throughput is reached.

The total power allocated to users is given by:

$$
P_{T}=\sum_{k=1}^{K} \sum_{n=1}^{N} p_{k, n} \cdot \rho_{k, n}
$$

The total bits for the kth user is given by:

$$
r_{k}=\sum_{n=1}^{N} c_{k, n} \cdot \rho_{k, n}
$$


where $\rho_{k, n}=1$ if $n t h$ sub-carrier is allocated to $k t h$ user and $\rho_{k, n}=0$ otherwise.(we consider that $n t h$ sub-carrier is allocated only to $k$ th user )

The problem of resource allocation can be written:

$$
\min \left(\sum_{k=1}^{K} \sum_{n=1}^{N} p_{k, n} . \rho_{k, n}\right)
$$

subject to:

$$
\sum_{n=1}^{N} c_{k, n} \cdot \rho_{k, n}>r_{0}
$$

where $r_{0}$ is the minimal data rate for the $k t h$ user.

\section{Formulation of the rate adaptive's problem}

Let's consider $\mathrm{N}$ and $\mathrm{K}$ respectively the total number of sub-carriers and the total number of the active users in the cell. The access method is based on the OFDMA and each user can have $N_{k}$ sub-carriers. According to Shannon's theorem [15], the capacity of the sub-carrier $n$ for the $k t h$ user is given by:

$$
C_{k, n}=\frac{B}{N} \log _{2}\left(1+S N R_{k, n}\right)
$$

where $B$ is the total bandwidth and $S N R_{k, n}$ the signal-tonoise ratio for the sub-carrier $n$. Let's consider $H_{k, n}$ the gain of the channel for the kth user on the nth sub-carrier. The signal to noise ratio for the $k t h$ user with the $n t h$ sub-carrier is defined by:

$$
S N R_{k, n}=\frac{P r_{k, n}}{N_{0} B_{c h}}
$$

where $P r_{k, n}$ is the power of the received signal, $N_{0}$ is the power spectral density of the noise and $B_{c h}$ is the width of one sub-channel. In this work, we assume that all sub-channels are flat during the transmission of the symbol and have the same width. We take into account the conditions of propagation characterized by the path-loss and the shadowing. Therefore, according to the equation of FRIIS ${ }^{1}$, the power of the received signal is given by:

$$
\operatorname{Pr}_{k, n}=\frac{P e_{k, n} H_{k, n}^{2} G e G r}{P_{L}}
$$

where $H_{k, n}, G e, G r$, and $P_{L}$ are respectively the gain of channel for user $k$ on the $n t h$ sub-carrier, the gain of the transmitter antenna, the gain of the receiver antenna and the path-loss factor. The path-loss factor is defined by:

$$
P_{L}=P_{L 0}+\sigma
$$

where $P_{L 0}$ is the free space path-loss and $\sigma$ is the term of shadowing. The path-loss factor is defined in three cases: the indoor radio propagation model, the outdoor to indoor and pedestrian environment and the vehicular environment.

Let's consider $R$ the distance expressed in $(\mathrm{km})$ between the mobile equipment and the base station, $n$ the number of floors in the path and $f_{n}$ the frequency of the transmission

\footnotetext{
${ }^{1}$ Harald T. Friis 1893-1976
}

carrier in $(M H z)$. In the case of indoor office radio propagation model, the path-loss is based on the COST 231 model. It's given in typical conditions by [8]:

$$
P L_{\text {indoor }}=37+30 \log _{10}(R)+18.3 n^{\left(\frac{n+2}{n+1}-0.46\right)}
$$

The path-loss in the outdoor to indoor and pedestrian propagation is the total transmission loss taking into account the reflexion and the diffraction. This path-loss is given by:

$$
P L_{\text {outdoor }}=49+40 \log _{10}(R)+30 \log _{10}\left(f_{n}\right)
$$

In the vehicular test environment the path-loss factor is defined by:

$$
\begin{array}{r}
P L_{\text {vehicular }}=\left[40\left(1-4.10^{-3} \Delta h_{b}\right)\right] \log _{10}(R) \\
-18 \log _{10}\left(\Delta h_{b}\right)+21 \log _{10}\left(f_{n}\right)+80
\end{array}
$$

The total throughput can be expressed by:

$$
C_{T}=\sum_{k=1}^{K} \sum_{n=1}^{N} C_{k, n} \cdot \rho_{k, n}
$$

where $\rho_{k, n}$ is the indicator of the resources allocation such that $\rho_{k, n}=1$ if the $n t h$ sub-carrier is attributed to the $k t h$ user and $\rho_{k, n}=0$ in otherwise.

By replacing (5) into (3), the total throughput can be written:

$$
C_{T}=\sum_{k=1}^{K} \sum_{n=1}^{N} \frac{B}{N} \log _{2}\left(1+\frac{P e_{k, n} H_{k, n}^{2} G e G r}{P_{L} N_{0} B_{c h}}\right) \cdot \rho_{k, n}
$$

Therefore, the main objective of this work is to maximize the total throughput under the constraints of the available power. Indeed, the capacity is proportional with the power of signal $P e_{k, n}$. Thus, the research of the maximization of the capacity is necessarily obtained with the increase of the power transmitted signal. So, the resolution of this problem is limited by the availability of the total power in the base station.

In this work, we propose to increase the signal-to-noise ratio by applying an efficient resources allocation to users. In fact, the sub-carriers are allocated to users with highest channel gain, in this case the capacity of the system is increased. The power on each sub-carrier is assumed to be constant.

The resources allocation problem can be formulated as:

$$
\max \left(\sum_{k=1}^{K} \sum_{n=1}^{N} \frac{B}{N} \log _{2}\left(1+\frac{P e_{k, n} H_{k, n}^{2} G e G r}{P_{L} N_{0} B_{c h}}\right) \cdot \rho_{k, n}\right)
$$

subject to:

$$
\begin{gathered}
\sum_{k=1}^{K} \sum_{n=1}^{N} P e_{k, n} \leq P_{T} \\
\sum_{k=1}^{K} \rho_{k, n}=1
\end{gathered}
$$

where $P_{T}$ is the total power available in the base station.

The problem described above is highly non linear and there are not an efficient algorithm which gives exact solution. 
Therefore, many algorithms are proposed to solve approximately the resources allocation problem. There are the iterative algorithms, and the heuristic approach.

The iterative approach is more simple to implement but its complexity increases highly when the parameters of the problem become very important. In addition, when the problem presents several extrema the iterative algorithms are not appropriated. Indeed, in this situation an iterative approach can converge to a local optimum which is not necessarily the global solution.

In otherwise, the heuristic approach, inspired by natural phenomenon, presents a very high robustness; which permits to efficiently solve the resources allocation problem even if it presents many extrema[6]. The evolutionary algorithm is one part of heuristic approach. The algorithm proposed in this work is based on genetic algorithms inspired by the evolution of species introduced by C. Darwin ${ }^{2}$.

\section{Genetic Algorithms}

Genetic algorithms are inspired by Darwin's theory of evolution and by Mendel's works about recombination of species[10]. GAs are used to solve many problems of optimization.

Robustness is the main advantage of genetic algorithms relative to traditional resolution methods of optimization problems[6]. In other words, we can see the four major differences between the two methods:

- GAs work with a coding of the set of parameters, while the classical methods use directly the parameters.

- The solution given by GAs is a set of points (chromosomes) and the solution for a classical methods is a single point.

- GAs use the objective function and the standards methods often use derivatives of function or other auxiliary knowledge.

- Gas use probabilistic transition rules when the traditional methods use deterministic rules.

The principle of GAs is based on the evolution of an initial population under the effect of operators such as selection, mutation and crossover. At the end of the GAs's process, the best individual in the population will be the solution of the optimization problem.

The different phases of the GAs are:

- Coding of chromosome: A chromosome of the population represents a resource allocation scheme. The coding of the chromosome is to provide a structure corresponding to the resource allocation problem. In our implementation, chromosome is represented by an array of structure, containing a fixed number of subcarriers, numbered in increasing order, from 0 to $N-1$. In the $n t h$ cell, there are the index of the user to which the corresponding subcarrier is allocated. The required power and the quantity of bits are calculated from this allocation for every user. All chromosomes have the same fixed size which corresponds to the total number of subcarriers. The table I shows an example of the structure of the chromosomes.

TABLE I. Structure of Chromosome

\begin{tabular}{|l|l|l|l|l|}
\hline Subcarrier & 1 & 2 & $\cdots$ & $\mathrm{N}$ \\
\hline User & 5 & 3 & $-\cdots$ & 10 \\
\hline
\end{tabular}

- Initialization of the population: It consists to set the size of the population and to generate all chromosomes of the population. In this work, the chromosomes of the population are randomly generated and their size is the same and stays constant during the GAs process.

- Evaluation: In this function, the total required power and the total throughput are calculated for each chromosome. The required power corresponds to a fitness (objective function) of the chromosome. The fitness or the objective function represents the criterion of the selection of chromosomes.

- Selection: After evaluating the power of all chromosomes, the selection consists to retain the best chromosome by directly sorting (natural selection) or by organizing tournament between two chromosomes arbitrary selected (selection by tournament). The best chromosome of the population is the one that the requires power is the smallest while respecting the constraint of throughput.

- Mutation: It consists to bring changes to the resources allocation scheme in order to have a better exploration of the search domain. In this work, the number of mutations and the index of the mutated subcarrier are randomly determined for each chromosome. Note that, all chromosomes will not be affected by the mutation.

- Crossover: In this step, a new chromosome is created from two chromosomes in order to take advantages of their best characters. Two points of crossover are randomly chosen and two parts of the first chromosome are concatenated with one part of the second chromosome. So, a new chromosome is created from this concatenation.

\section{DESCRIPTIONS OF PROPOSED SOLUTIONS}

The genetic algorithms is among the evolutionary approaches. It's first proposed in engineering by J. Holland ${ }^{3}$ and developed by David Goldberg [6] for solving the problem of the control of natural gas pipeline. The principle of genetic algorithms is based on the evolution of the initial population by many factors such as: selection, crossover, recombination and mutation. At the end of the process, a new population is created. These operations are repeated during the cycle of evolution defined by the number of generations.

${ }^{3}$ John Henry Holland, 1929, University of Michigan 


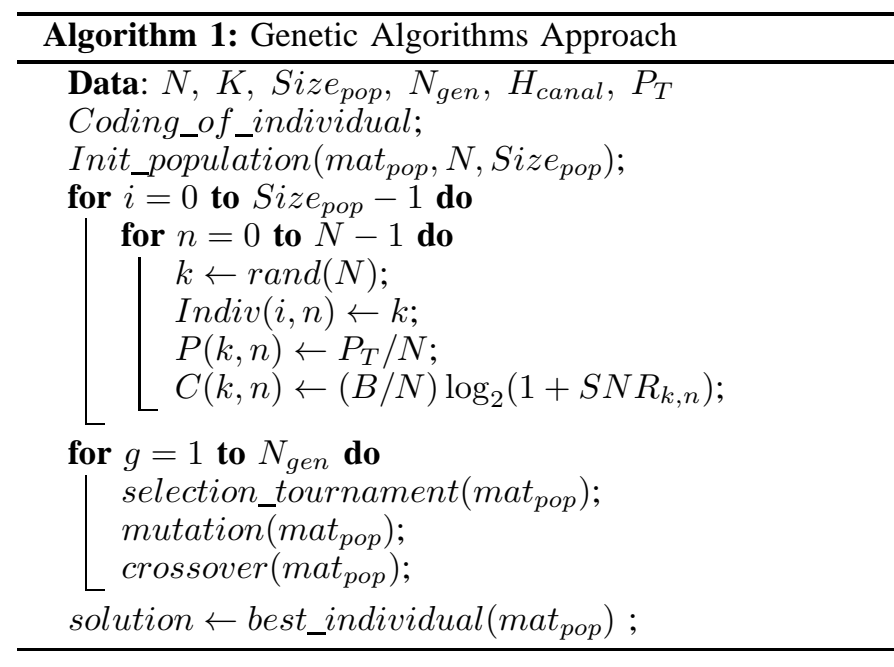

\section{Simulation AND RESUlts}

\section{A. Parameters of simulation}

In figure 3, the model of transmission channel is shown. It's characterized by frequency and time selectivity which take into account the multi-path phenomenon and the Doppler effect. The Jakes' model is used for the simulation of the channel with the parameters given in the table II.

Figure 3 shows that according to the considered frequency, the gain of the transmission channel can vary from $15 \mathrm{~dB}$ to -25 $\mathrm{dB}$. This reflects the frequency selectivity of the transmission channel. Note that, this channel gain for a given frequency also varies from one moment to another, reflecting the temporal selectivity of the transmission channel.

TABLE II. PARAMETERS OF SIMULATION

\begin{tabular}{|l|l|}
\hline Channel bandwidth & $20 \mathrm{MHz}$ \\
\hline PSD of the noise $N_{0}$ & $-174 \mathrm{dBm} / \mathrm{Hz}$ \\
\hline Sub-channel bandwidth & $15 \mathrm{kHz}$ \\
\hline Total power of BS & $30 \mathrm{Watts}$ \\
\hline Radius of cell & $2 \mathrm{~km}$ \\
\hline RMS of delay & $150 \mathrm{~ns}$ \\
\hline Average speed of users & $1 \mathrm{~m} / \mathrm{s}$ \\
\hline Numbers of scatters & 10 \\
\hline Base Station antenna gain & $15 \mathrm{dBi}$ \\
\hline Mobile Station antenna gain & $0 \mathrm{dBi}$ \\
\hline
\end{tabular}

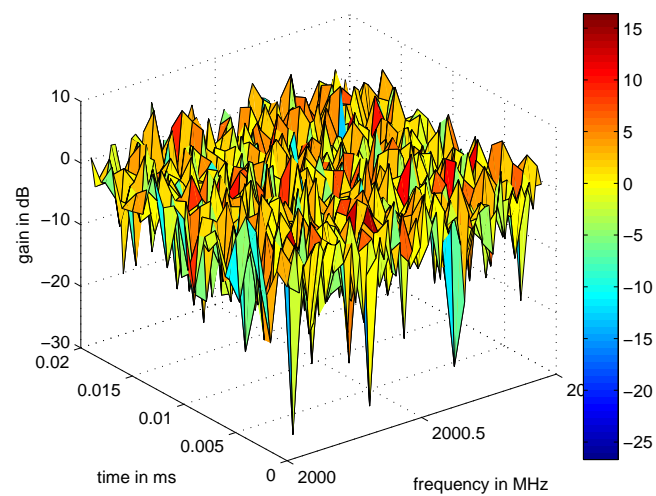

Fig. 3. Variation of channel gain in frequency and time domain

\section{B. Results of simulation}

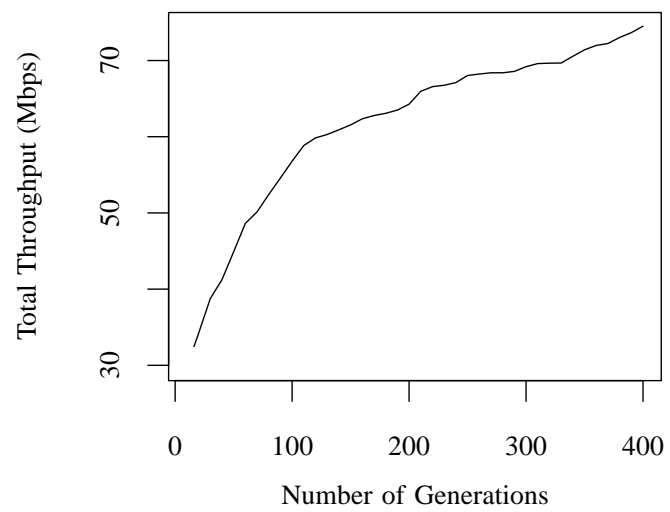

Fig. 4. Total Throughput Vs Nb. generations

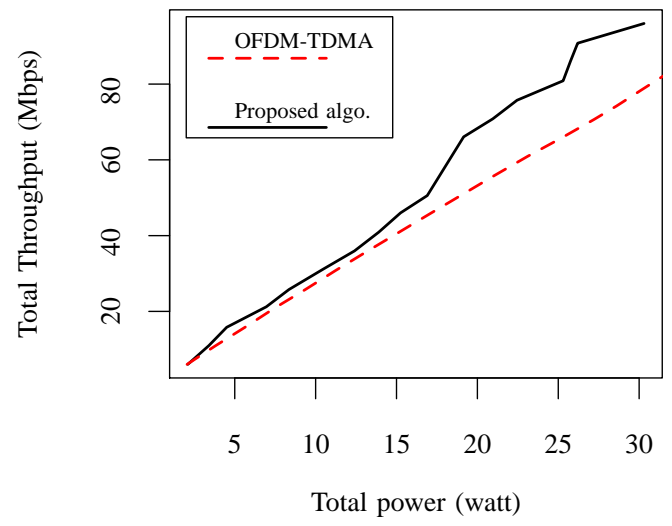

Fig. 5. Total Throughput Vs Total power

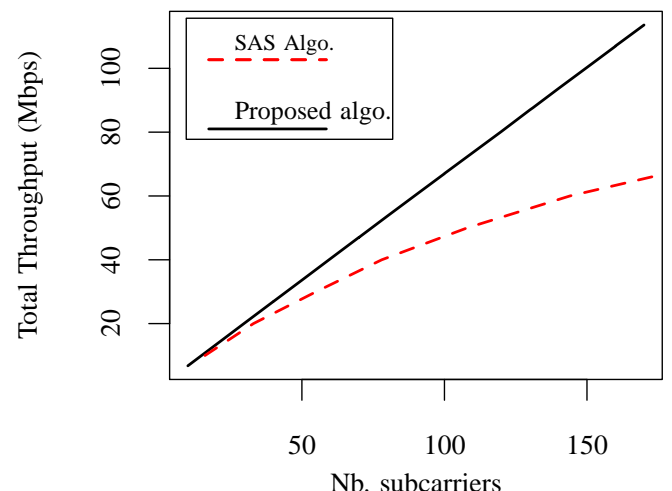

Fig. 6. Total Throughput Vs Nb. subcarriers

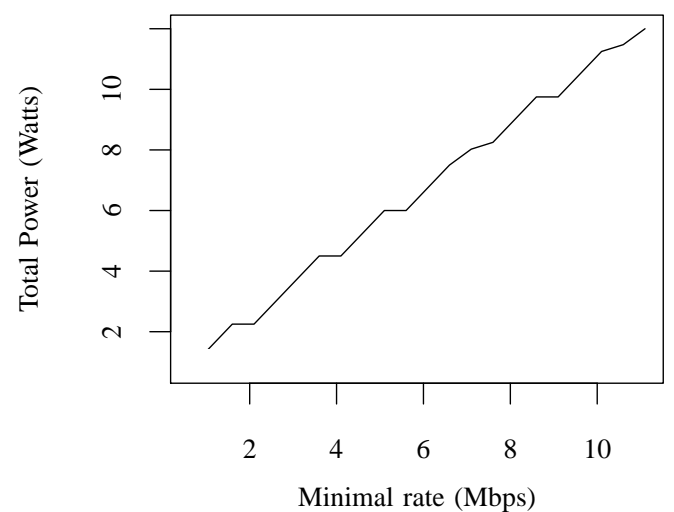

Fig. 7. Total power vs minimal users Throughput 


\section{Analysis and comments of results}

The results obtained with the proposed algorithm are very interesting. Indeed, figure 4 shows the convergence of the proposed method. We note a quick increase of the total throughput when the number of generation grows. However, we note that from the 120th generation, the acceleration of the graph decreases. That corresponds to the neighboring of the optimal solution of the problem given by the proposed method.

In figure 5, we compare the proposed algorithm with the OFDM-TDMA approach. Figure 5 shows that, the proposed algorithm performs the OFDM-TDMA algorithm; and this improvement becomes very important for high data rate. For exmple with power consumption of $25 \mathrm{w}$, the proposed algorithm permits to acheive around $80 \mathrm{Mbps}$ while the OFDMTDMA gives $60 \mathrm{Mbps}$.

Also, in Figure 6, we compare the proposed algorithm with the SAS (Simulated Annealing) algorithm proposed in [4]. We note that, for average flows (about less than 50Mbps), the results of our approach are similar to those obtained with SAS algorithm. However, when the required flow rate becomes large(from $60 \mathrm{Mbps}$ ), the gain of the proposed solution is more pronounced relative to the SAS algorithm. Sometimes, the proposed approach provides similar flow with more than half of number of sub-carriers used by the SAS method. As an exemple, the proposed algorithm gives $100 \mathrm{Mbps}$ with 150 sub-carriers while the SAS method does not exceed $60 \mathrm{Mbps}$ with more than 150 sub-carriers.

Figure 7 shows the evolution of the users's consumed power based on the minimum required data rate for users. We note that, the consumed power does not evolve linearly with the required users's throughput. Better, figure 7 shows a decrease in the slope of the curve of evolution when the users's throughput increases. This reflects a minimization of the power consumption depending on the data rate.

\section{CONCLUSION}

In this paper, we have proposed the genetic algorithms to solve the problem of resources allocation. The study took place in the context of frequency selectivity and time-variant of the transmission channel. In addition, we have taken into account the propagation loss; which permits to obtain the effective received throughput for users. The proposed implementation of genetic algorithms has led to efficiently solve the problem of resources allocation, and it's better than the OFDM-TDMA approach and the algorithm SAS. The obtained solution is very similar to the standard specifications of OFDMA-based systems in terms of maximum data rate and capacity of users. In this article, the choice of the methods of comparison with our approach is firstly justified by the good performance of some approaches: it's the case of SAS algorithm [4], on the other hand by the accessibility of certain resolution methods found in the literature: it's the case of OFDM-TDMA approach. On the other side, comparison with methods based on evolutionary approach is made difficult by the lack of details on the algorithms presented in most of the articles that we consulted [1][12][17].

However, the proposed method solves the problem of resources allocation in mono-cell case. We hope that this approach will give interesting results when applied to the case of multi-cell system and in radio cognitive systems. In future work, the integration of new metric of QoS measurement of cellular systems such as an effective spectral efficiency area [9], will constitute the basis of a new development in order to achieve a more effective resolution of the resources allocation problem in radio mobile systems.

\section{REFERENCES}

[1] H. Ahmadi and Y. Chew. Adaptive subcarrier-and-bit allocation in multiclass multiuser ofdm systems using genetic algorithm. 2009.

[2] M. Alouini and A. Goldsmith. Adaptive m-qam modulation over nakagami fading channels. Proc. IEEE. Global Communication Conf., pages 218-223, 1997.

[3] Q. An and Y. Yang. Efficient water-filling algorithm for power allocation in ofdm-based cognitive radio systems. CSE Conference and Workshop Papers, (196), Janvier 2012.

[4] J. Farah and F. Marx. Greedy algorithms for spectrum management in ofdm cognitive systems - applications to video streaming and wireless sensor networks. IntechOpen, November 2008.

[5] S. Pleftschinger G. Münz and J. Speidel. An efficient waterfilling algorirhm for multiple access ofdm. Stuttgart Germany, 2002.

[6] David E. Goldberg. Genetic Algorithms in Search, Optimization and Machine Learning. MA Addison Wesley, USA, 1th edition, 1989.

[7] T. Ibaraki and N. Katoh. Resource Allocation Problems-Algorithmic Approches. MIT Press, Cambridge MA USA, 1988.

[8] ITU. Guidelines for evaluation of radio transmision technologies for imt-2000. Technical report, International Telecommunication Union, Place des Nations 1211 Geneva 20 Switzerland, 1997.

[9] A. Omri M. O. Hasna and M. Nafie. Effective area spectral efficiency for wireless communication networks with interference management. EURASIP Journal on Wireless Communications and Networking, August 2015.

[10] Mélanie Mitchell. An Introduction to Genetic Algorithms. MIT Press, USA, 1th edition, 1999.

[11] John G. Proaski. Digital Communications. McGraw Hill, New York USA, 4th edition, 2001.

[12] Y. Reddy and N. Gajendar. Evolutionary aproach for efficient resource allocation in multi-user ofdm systems. Las Vegas Nevada USA, 2007.

[13] Hermann Rohling. OFDM: Concepts for Future Communication Systems. Springer, Hamburg Germany, 2011.

[14] Henrik Schulze and Christian Lüders. Theory and Apllication of OFDM and CDMA. John Wiley \& Sons Ltd, Germany, 1th edition, 2005.

[15] Claude E. Shannon. Communication in the presence of noise. volume 86, February 1998.

[16] J. C. Tu and J. M. Cioffi. A loading algorithm for the concatenation of coset codes with multichannel modulation method. IEEE GLOBECOM, December 1990.

[17] N. Zhou X. Zhu and Y. Huang. Genetic algorithms based crosslayer resource allocation for wireless ofdm networks with heterogeneous traffic. volume 6, pages 1656-1659, Glasgow Scotland, Aug. 2001. 